\title{
Appearance concern and depression in adolescent girls with systemic lupus erythematous
}

\author{
Li Ji • Shi Lili • Wei Jing • He Yanyan • Wei Min • \\ Xiao Juan • Song Hongmei
}

Received: 17 April 2012 / Revised: 6 August 2012 / Accepted: 10 August 2012 / Published online: 9 September 2012

(C) Clinical Rheumatology 2012

\begin{abstract}
To examine the relationship between physical appearance concern and psychological distress in female adolescent patients with systemic lupus erythematosus (SLE) was the aim of this study. A total of 84 adolescent SLE female patients and 80 age-matching healthy adolescents were evaluated for levels of appearance concern and a range of illness-specific measures to determine how these demographic and clinical variables were related to the dependent variable psychological distress. The Systemic Lupus Erythematosus Disease Activity Index was used to assess disease activity. Assessment of depression was conducted through the children depression inventory (CDI). Appearance concern was measured using the SelfPerception Profile for Children. The total CDI was $18.5 \pm$ 4.3 , indicating that these patients had more depressive symptoms, comparing with age- and sex-matched healthy controls. Furthermore, a total of 32 (38.1\%) patients had CDI larger than 19 points, indicating that they have relatively higher risk in developing depression. The CDI in control group was significantly lower than that in the SLE group $(11.4 \pm 1.7$ vs. $18.5 \pm 4.3, t=9.93, p<0.05)$. Using correlation and multiple regression analyses, we found that both appearance concern and age were predictive of depression in patients with SLE, and the former was highly correlated. This indicates that appearance concern may be associated with depression in female adolescent SLE
\end{abstract}

L. Ji $\cdot$ H. Yanyan $\cdot$ W. Min $\cdot$ X. Juan $\cdot$ S. Hongmei $(\bowtie)$ Pediatrics Department, Peking Union Medical College Hospital, Peking Union Medical College, Chinese Academy of Medical Sciences,

Beijing, China 100730

e-mail: pumc.dr.song@gmail.com

S. Lili $\cdot$ W. Jing

Psychology Department, Peking Union Medical College Hospital, Peking Union Medical College, Chinese Academy of Medical Sciences,

Beijing, China patients. The results suggest that appearance concern is strongly associated with depression in female adolescent patients with SLE and should be routinely assessed.

Keywords Adolescent · Appearance · Depression · Systemic lupus erythematosus

\section{Introduction}

Systemic lupus erythematous (SLE) is a chronic inflammatory autoimmune disorder that mainly affects multiple organ systems. The onset of SLE occurs in childhood or adolescence and accounts for around $15 \%$ of affected individuals [1]. Young persons with SLE generally have severe disease at onset, requiring high-dose corticosteroid and immunosuppressive drugs for disease control [2]. In children, SLE is associated with significant morbidity consequent to the disease $[3,4]$. The treatment of SLE results in a wide-ranging spectrum of physical, functional, and psychological damage that impacts the well-being of children with SLE. The combination of active disease and side effects of corticosteroid treatment, such as weight gain, facial rashes and physical inability, hirsutism, striae, and hair loss, may result in difficulties in daily life for adolescents with SLE.

Also, SLE and the corticosteroid treatment could lead to high rates of disturbance to body image. Adolescents are still in the critical physical and mental development period and are very sensitive to their appearance change and the opinions from other people. Therefore, they are more vulnerable to the psychological distress and may develop depression more easily, comparing with the adult patients. Body dissatisfaction is associated with emotional distress and low sense of self-value [5, 6]. It has been demonstrated that women with rheumatoid arthritis have lower levels of self-esteem compared with healthy women [7] and high levels of body image dissatisfaction [8]. Although SLE is 
associated with perceptible changes to physical appearance, body image concern have received little empirical investigation, especially in the pediatric population. With this background, the aim of the present study was to investigate the relationship between appearance and psychological distress in a Chinese cohort of adolescent affected with SLE.

\section{Materials and methods}

\section{Participants}

Patients age 10-18 with a definite diagnosis of SLE who were followed and hospitalized at the Pediatrics Department, Peking Union Medical College Hospital were recruited. Patients were excluded if they had been diagnosed within the preceding 3 months, were $>18$ years of age at diagnosis, or had difficulties in understanding and completing the questionnaires. Age- and sex-matched healthy controls were recruited from a nearby middle school. Study assessments occurred between April 2010 and December 2011. All caregivers and adolescents provided assent and consent prior to participation. Ethical approval was obtained from the Peking Union Medical College Hospital's Clinical Research Ethics Board and the hospital's Research Review Committee.

\section{Methods}

Study participants underwent a clinical assessment by a pediatric rheumatologist in hospitalization period. Assessment included a complete physical examination and data collection for age, sex, disease duration, number of admissions, and current medications. After the initial assessment, they were required to finish questionnaires and could raise questions with the physicians if they encountered any problems. Meanwhile, confidentiality and privacy were assured from both physicians and parents

\section{Disease measures}

The SLE diagnosis is based on the criteria for the diagnosis of SLE revised by the American College of Rheumatology in 1997 [9]. The Systemic Lupus Erythematosus Disease Activity Index (SLEDAI) [10] was used to assess disease activity. The SLEDAI assesses 24 clinical and laboratory measures of SLE activity with a score range of $0-105$; a higher score corresponds to higher disease activity. The following activity categories have been defined on the basis of SLEDAI scores: no activity (SLEDAI $=0-4)$, mild activity (SLEDAI $=5-9)$, moderate activity (SLEDAI $=10$ 14 ), and high activity (SLEDAI $\geq 15$ ) [11].
Questionnaires

Assessment of depression was conducted through the children depression inventory (CDI) [12]. The CDI is a brief self-report test that helps assess cognitive, affective, and behavioral signs of depression in children and adolescents 7-18 years old. It contains 27 items; for each item, the child has three possible answers: 0 indicating an absence of symptoms, 1 indicating mild symptoms, and 2, definite symptoms. Total scores range from 0 to 54, and Kovacs [13] indicates a 19-point cutoff as the ideal threshold discriminating children at risk of depression from nondepressed children [14]. The CDI generally covers negative mood, interpersonal difficulties, negative self-esteem, ineffectiveness, and inability to experience pleasure. For each item, the individual is asked to select the statement that best describes his or her feelings for the past 2 weeks. The corresponding questionnaires in Chinese version have been validated among Chinese subjects with good reliability and validity [15].

Appearance concern was assessed by the physical appearance domain of the multidimensional Self-Perception Profile for Children (SPPC) [16]. Six items (Likert scale 1-4) were used to assess the youths' perceived competence in physical appearance with questions such as whether my appearance make me feel upset and whether my hair looks beautiful. The internal reliability in this sample for physical appearance domain was $\alpha=0.85$. Evidence for the validity for the SPPC and the specific domains of competence has been reported in numerous studies and has been employed in numerous studies in the developmental and social developmental literature [17]. Moreover, lowered levels of SPPCassessed self-esteem have been found in clinically referred children and adolescents suffering from disruptive and emotional disorders [18].

Statistical analysis

Statistical analysis was performed using the Statistical Package for the Social Sciences (SPSS for Windows v. 11.0, Chicago, IL, USA). An outcome was considered statistically significant if $p<0.05$. A $t$ test was used to compare the data between the SLE patients and controls. Correlation and multiple regression analyses were used to analyze the data. Correlation coefficients were defined as follows: 0 0.25 , negligible or not correlated; $0.25-0.50$, fair correlation; $0.50-0.75$, moderate-to-good correlation; and $>0.75$, very good-to-excellent correlation. All independent variables were entered into the SPSS to determine the degree to which these accounted for variance in depression: age, disease duration, SLEDAI, number of admissions, and appearance concern were entered. 


\section{Results}

Demographic and clinical characteristics

Of the 84 SLE patients and 80 age- and sex-matched healthy controls that were approached, all returned a completed questionnaire. Table 1 lists the subjects' demographics and clinical characteristics. The SLE patients had an average of $3.6 \pm 1.2$ hospital admissions and the prednisone dose ranged from 15 to $60 \mathrm{mg} /$ day. All patients were taking prednisone, and $57(67.9 \%)$ were taking at least one non-corticosteroid immunosuppressant. Disease activity and disease damage were variable as indicated by SLEDAI.

\section{CDI}

Nearly all patients showed increased depressive symptoms as indicated by the mean scores on the CDI. The total mean CDI was $18.5 \pm 4.3$, indicating that these patients had more depressive symptoms, comparing with age- and sexmatched healthy controls. Furthermore, a total of 32 (38.1\%) patients had CDI larger than 19 points. The CDI in control group was significantly lower $(11.4 \pm 1.7$ vs. $18.5 \pm 4.3$, $t=9.93, p<0.05$ ), and no one in the control group had a CDI score over 19. Among the CDI subscales, the negative mood, negative self-esteem, and anhedonia contributed to the total scores mostly.

\section{Analyzes}

With regard to concern about appearance, 77 (91.7\%) patients with SLE reported that they felt unattractive due to the disease according to the questions from the SPPC. Among the SLE patients, the SPPC physical appearance score was $13 \pm 2.8$, while the score of control group was

Table 1 Demographic and clinical background

\begin{tabular}{lll}
\hline Characteristics & SLE patients & Control \\
\hline No. of subjects & 84 & 80 \\
Child per family & $1.3 \pm 0.5$ & $1.1 \pm 0.7$ \\
Age (years) & $15.3 \pm 1.4$ & $15.7 \pm 1.5$ \\
Disease duration (months) & $15.8 \pm 7.9$ & N/A \\
No. of admissions & $3.6 \pm 1.2$ & N/A \\
SLEDAI & $7.9 \pm 4.0$ & N/A \\
No activity (\%) & $22.6 \%$ & N/A \\
Mild activity (\%) & $41.7 \%$ & N/A \\
Moderate activity (\%) & $25.0 \%$ & N/A \\
High activity (\%) & $10.6 \%$ & N/A \\
SPPC physical appearance score & $13 \pm 2.8$ & $20 \pm 3.1$ \\
CDI & $18.5 \pm 4.3$ & $11.4 \pm 1.7$ \\
\hline
\end{tabular}

$20 \pm 3.1(t=11.71, p<0.05)$, indicating that the SLE adolescents believe their appearances were severely impaired.

Univariate Pearson correlations were computed between physical age, disease duration, SLEDAI, number of admissions and appearance concern, and CDI. Appearance concern was highly correlated with the depression $(r=0.758, p=$ $0.001)$. Subsequently, age was moderately correlated with depression $(r=0.468, p=0.001)$. Other variables, such as number of admissions, SLEDAI, and disease duration, were partially correlated with depression. The detailed correlations are presented in Table 2. A multiple regression analysis entering those significant variables shows: $\mathrm{CDI}=1.124 \times$ appearance concern $+0.091 \times$ SLEDAI $+0.019 \times$ age +1.831 $\left(R^{2}=0.587\right)$, which indicates that CDI could be largely estimated if appearance concern score is known, while SLEDAI and age would bring the estimation close to the accurate value.

\section{Discussion}

Among the many difficulties faced by female adolescents with SLE is the distress associated with temporary or permanent changes to appearance resulting from disease and treatment. Experiencing these changes during adolescence presents particular challenges as the usual processes of physical, social, and sexual development at this time often results in individuals being acutely aware of their body [19]. This also could explain why older adolescents showed more depressive symptoms in this study. Physical appearance is central to youth culture and contributes more than any other factor to levels of overall self-esteem in the normal adolescent population [20]. Very little research has focused specifically on the stress of an altered appearance during SLE treatment. What evidence there is, combined with research investigating more generic experiences of adolescents living with SLE, suggests that an altered appearance during treatment can bring with it a host of psychosocial consequences that affect quality of life.

In this study, we find that in patients with SLE, appearance concern was related to depression. A study enrolling 1,685 children and adolescents, using CDI as indicators for depressive symptoms in China, had concluded that the

Table 2 Univariate correlations of variables with CDI

\begin{tabular}{llc}
\hline Items & $r$ & $p$ value \\
\hline Age & 0.468 & 0.001 \\
Disease duration & 0.054 & 0.626 \\
Number of admissions & 0.272 & 0.012 \\
SLEDAI & 0.180 & 0.101 \\
Appearance concern & 0.758 & 0.001 \\
\hline
\end{tabular}


average total score of CDI were $10.75 \pm 6.73$ for boys and10.16 \pm 6.37 for girls, respectively [15]. However, the average CDI of patients in SLE group was $18.5 \pm 4.3$, which was significantly higher than the normative value. In the 84 patients, $32(38.1 \%)$ patients had $\mathrm{CDI}>19$ points, meaning that these patients were at extremely high risk of developing depressive symptoms, at least based on the hospitalization circumstance. Benrud-Larson also found that appearance was predictive of depression in adult female patients with rheumatic diseases. Individuals with concern regarding their physical appearance reported more symptoms of depression. This result is similar to those found in studies of other rheumatic diseases [21] and confirms the link between appearance concern and depression in patients with rheumatic disease.

Our results clearly establish that appearance concern may be associated with depression in adolescent patients with SLE. Although body image dissatisfaction may be predictive of depression, this does not establish a causal relationship. It is also possible that depression causes body image dissatisfaction, rather than dissatisfaction causing depression. To date, there is no prospective research to confirm the direction of causality. It is likely that the relationship is reciprocal, that is, depression resulting from the disease increases appearance concern and dissatisfaction intensifies feelings of depression. It is not possible to determine from this study the direction of the relationship between appearance concern and depression.

However, there are several limitations in this study. A limitation of this study was the use of a generic measure for physical appearance evaluation. Although the SPPC has been used in varieties of pediatric populations, it is less robust than a more comprehensive one and less effective than a SLE-specific evaluation tool. This is largely unavoidable because there are no well-validated measures of appearance that have been extensively used with patients with rheumatic diseases. However, future research could work to develop a rheumatology-specific measure of appearance concern. Secondly, it is worth noting that the selfadministered evaluation of depression through the CDI may fail to capture the whole depressive disorders, though the CDI has indicated the high prevalence of depressive symptoms. This finding further highlights the importance of a deeper evaluation of affective disorders in children and adolescents, such as the use of structured interviews. Thirdly, we failed to evaluate appearance concern and psychological conditions of the adolescents before the SLE onset because the patients who received these questionnaires had been treated with an average of 15.8 months. However, in the future study, we may develop parents administered questionnaires to indirectly evaluate the patient's condition before the disease onset. Lastly, we did not recruit boys in this study because of the low incidence in male population; however, we may continue collecting male adolescent patients data in the future study.

It is important to understand the psychological distress in adolescent SLE patients. Because adolescent SLE patients are in a stage when physical and psychological developments are in rapid development, they are more concerned to their appearance, especially in front of the opposite sex. Some girls refuse corticosteroid administration due to the treatment-related side effects, such as obesity, but result in severe SLE relapse; some girls deliberately and quietly lower the medication dose to avoid side effects but bring more side effects. Additionally, most of the children live in one-child family; therefore, they are tended to be more vulnerable when they do not have siblings. Of course, even if a young person is an only child, if they have a good friendship circle, this can reduce problems around social isolation. In this circumstance, psychological support is more necessary, and the clinicians involved in the SLE care will need to be more involved with the children inner mind and family problems. Meanwhile, psychosocial screening should be routinely performed by health professionals in everyday practice to screen out the psychologically compromised adolescents [22, 23].

Acknowledgments We are grateful to the physicians, nurses, the patient's caregivers, and psychologist of the Peking Union Medical College Hospital, Beijing, China for facilitating this project. We thank Dr. Zhang Xiaowei, Urology Department, Peking University People's Hospital, and Dr. Song Qingkun, Department of Public Health, Chinese University of Hong Kong, Hong Kong for expert statistical advice and manuscript revision. Lastly, we want to specially thank Dr. Wang Qian, Rheumatology, Peking Union Medical College Hospital, for guidance and cooperation in data collection.

Disclosures None.

\section{References}

1. Watson L, Leone V, Pilkington C et al (2012) Juvenile-onset SLE; disease activity, severity and damage - the UK JSLE cohort study. Arthritis Rheum. doi:10.1002/art.34410

2. Beresford MW, Davidson JE (2006) Adolescent development and SLE. Best Pract Res Clin Rheumatol 20:353-368

3. Ravelli A, Ruperto N, Martini A (2005) Outcome in juvenile onset systemic lupus erythematosus. Curr Opin Rheumatol 17:568-573

4. Ruperto N, Buratti S, Duarte-Salazar C et al (2004) Health-related quality of life in juvenile-onset systemic lupus erythematosus and its relationship to disease activity and damage. Arthritis Rheum $51: 458-464$

5. Chen H, Jackson T, Huang X (2006) The negative physical self scale: initial development and validation in samples of Chinese adolescents and young adults. Body Image 3:401-412

6. Hoffman JM, Brownell KD (1997) Sex differences in the relationship of body fat distribution with psychosocial variables. Int J Eat Disord 22:139-145

7. Skevington SM, Blackwell F, Britton NF (1987) Self-esteem and perception of attractiveness: an investigation of early rheumatoid arthritis. Br J Med Psychol 60(Pt 1):45-52 
8. Ben-Tovim DI, Walker MK (1995) Body image, disfigurement and disability. J Psychosom Res 39:283-291

9. Stoll T, Stucki G, Malik J, Pyke S, Isenberg DA (1997) Association of the Systemic Lupus International Collaborating Clinics/American College of Rheumatology Damage Index with measures of disease activity and health status in patients with systemic lupus erythematosus. J Rheumatol 24:309-313

10. Bombardier C, Gladman DD, Urowitz MB, Caron D, Chang CH (1992) Derivation of the SLEDAI. A disease activity index for lupus patients. The committee on prognosis studies in SLE. Arthritis Rheum 35:630-640

11. Gladman DD, Ibanez D, Urowitz MB (2002) Systemic Lupus Erythematosus Disease Activity Index 2000. J Rheumatol 29:288291

12. Knight D, Hensley VR, Waters B (1988) Validation of the children's depression scale and the children's depression inventory in a prepubertal sample. J Child Psychol Psychiatry 29:853-863

13. Kovacs M (1981) Rating scales to assess depression in schoolaged children. Acta Paedopsychiatr 46:305-315

14. Helsel WJ, Matson JL (1984) The assessment of depression in children: the internal structure of the child depression inventory (CDI). Behav Res Ther 22:289-298

15. Yu D, Xu L (2000) Preliminary use of the children's depression inventory in China. Zhong Guo Xin Li Wei Sheng Za Zhi 14:225-227
16. Harter S (1985) Manual for the self-perception profile for children. University of Denver, Denver

17. Hoare P, Mann H (1994) Self-esteem and behavioural adjustment in children with epilepsy and children with diabetes. J Psychosom Res 38:859-869

18. Dumas D, Pelletier L (1999) A study of self-perception in hyperactive children. MCN Am J Matern Child Nurs 24:12-19

19. Holmbeck GN (2002) A developmental perspective on adolescent health and illness: an introduction to the special issues. J Pediatr Psychol 27:409-416

20. Coleman JCHL (1999) The nature of adolescence, 3rd edn. Routledge, New York

21. Benrud-Larson LM, Heinberg LJ, Boling C et al (2003) Body image dissatisfaction among women with scleroderma: extent and relationship to psychosocial function. Health Psychol 22:130-139

22. Robertson LP, McDonagh JE, Southwood TR, Shaw KL (2006) Growing up and moving on. A multicentre UK audit of the transfer of adolescents with juvenile idiopathic arthritis from paediatric to adult centred care. Ann Rheum Dis 65:74-80

23. Yeo MS, Bond LM, Sawyer SM (2005) Health risk screening in adolescents: room for improvement in a tertiary inpatient setting. Med J Aust 183:427-429 